\title{
Equalization equations in reactant resolution
}

\author{
JACEK KORCHOWIEC \\ K Guminski Department of Theoretical Chemistry, Jagiellonian University, R Ingardena 3, 30-060 \\ Cracow, Poland \\ e-mail: korchow@chemia.uj.cdu.pl
}

\begin{abstract}
The chemical system can be analyzed in different resolutions. The assumed resolution imposes a given partitioning of the system in physical or functional space. The most frequently explored are global, reactant, atoms-in-molecule, orbital, and local resolutions. In this paper we have considered reactant resolution, i.e., the mutually polarized reactants before the charge-transfer among them. We have demonstrated that a certain type of generalized sensitivity, the system responses to the population variables, is equalized throughout the space up to the infinite order in the perturbation expansion.
\end{abstract}

Keywords. Chemical potential; hardness; equalization equations; reactivity descriptors.

\section{Introduction}

Density functional theory (DFT) ${ }^{1}$ has had enormous impact on modern chemistry. Even though the direct computational approaches ${ }^{2}$ have rather historical significance and cannot describe the chemical phenomena, the indirect Kohn-Sham (KS) ${ }^{3}$ formulation has become a dominant approach nowadays to electronic structure calculations. It is reflected in the growing number of publications that apply the KS method to describe the chemical reactions, especially when transition metals are involved. This undoubted success of DFT methods is connected with the electron correlation that is included in computationally not demanding way. Therefore, the scaling properties of KS type methods are much better than the post-Hartree-Fock methods and almost identical to (or even better than) non-correlated Hartree-Fock method.

Apart from the computational convenience, DFT was quickly recognized as a useful tool giving theoretical explanation to many intuitive chemical concepts and rules. ${ }^{4-7}$ Among them is the electronegativity, identified as the negative electron chemical potential. ${ }^{8,9}$ The electron chemical potential is a Lagrange multiplier for the electron density, $\rho(\mathbf{r})$, normalization in the stationary principle of DFT,

$$
\delta\left\{E_{v}[\rho]-\mu\left(\int \rho(\mathbf{r}) \mathrm{d} \mathbf{r}-N\right)\right\}=0,
$$

and is by definition equalized throughout the space

$$
\left.\mu \equiv\left[\delta E_{v} / \delta \rho(\mathbf{r})\right]_{v}\right|_{\rho_{\mathrm{eq}}},
$$

thus validating Sanderson's electronegativity equalization principle. ${ }^{5}$ The functional derivative of the system energy $E_{\mathrm{v}}$ with respect to the electron density $\rho(\mathbf{r})$ (2) is evaluated for equilibrium density, $\rho_{\text {eq. }}$. The only constraint is imposed on the external potential $v(\mathbf{r})$, i.e., the nuclei are in the fixed positions. The other, commonly discussed quantities are the chemical hardness and softness, especially in the framework of hard-soft-acids-and-bases (HSAB) principle. ${ }^{6,7}$ The former is the second-order derivative of the system energy with respect to the electron population variable $(N)$, while the latter is the inverse of the hardness. ${ }^{8,10}$

The quantities appearing in the second-order Taylor expansion of the system energy in global $E=E(N, v)$ or local $E=E(\rho, v)$ representation are often considered as a starting point for deriving different reactivity descriptors, e.g., the Fukui function (FF), ${ }^{8,11-14}$ regional analogous of HSAB principle, ${ }^{15}$ electrophilicity ${ }^{16}$ and philicity ${ }^{17}$ indices. Especially interesting is the local hardness which like to the chemical potential can be equalized throughout the space. ${ }^{18}$ However, due to ambiguity in its definition leading to the position dependence is often considered as convenient reactivity indicator. For more information the reader is referred to recent monograph of Geerlings and co-workers. ${ }^{13}$ Nevertheless, the highorder system responses to population or external potential perturbation are less explored. The hardness population derivative was discussed by Parr and Fuentealba. ${ }^{19}$ Nonlinear FFs and hardnesses up to arbitrary perturbation order, were analyzed by Senet. ${ }^{20}$ 
The works on higher order derivatives of nuclear FF should also be mentioned. ${ }^{21,22}$

In this paper we concentrate on high-order population derivatives of the system energy in reactant resolution (mutually polarized reactants before charge-transfer), namely on the corresponding equalization equations. First, the global equilibrium is briefly sketched out. Then, the inter-reactant equilibrium is considered. The equalization equations in global, as well as in reactant resolutions are proved using the induction principle. Finally, the conclusions are given.

\section{Global equilibrium}

Even though the chemical potential in the case of global equilibrium is equalized by definition (see (1)), we repeat here the proof, for the current needs, using the following chain rule transformation:

$$
\mu(\mathbf{r}) \equiv[\delta E / \delta \rho(\mathbf{r})]=\frac{\partial E}{\partial N} \frac{\delta N}{\delta \rho(\mathbf{r})}=\mu \int \frac{\delta \rho\left(\mathbf{r}^{\prime}\right)}{\delta \rho(\mathbf{r})} \mathrm{d} \mathbf{r}^{\prime}=\mu .
$$

Note that in the above equation we compute the chemical potential in local resolution but the derivative is evaluated for equilibrium density (not exactly specified in the equation). The local resolution corresponds to the totally constrained (frozen) electron density with all infinitesimal volume elements being mutually closed. Therefore, the perturbation at $\mathbf{r}$ differentiates the chemical potential from point to point $\left(\mu(\mathbf{r}) \neq \mu\left(\mathbf{r}^{\prime}\right) \neq \mu\left(\mathbf{r}^{\prime \prime}\right) \neq \ldots\right)$ and the global equilibrium can be restored by removing the constraints. The change of variables in $(3)(\partial / \partial N)(\delta N / \delta \rho))$ indicates such a removal of constraints. In other words, the functional derivative is evaluated for equilibrium electron density $\left(\rho_{\text {eq. }}\right)$. In the same fashion, one can prove that the second-order derivatives of the system energy with respect to population variables (hardnesses) are equalized, ${ }^{12,18}$

$$
\eta \equiv(\partial \mu / \partial N)_{v}=\eta(\mathbf{r}) \equiv[\delta \mu / \delta \rho(\mathbf{r})]_{\mathrm{v}}=[\partial \mu(\mathbf{r}) / \partial N],
$$

namely,

$$
\eta(\mathbf{r}) \equiv[\delta \mu / \delta \rho(\mathbf{r})]=\frac{\partial \mu}{\partial N} \frac{\delta N}{\delta \rho(\mathbf{r})}=\eta \int \frac{\delta \rho\left(\mathbf{r}^{\prime}\right)}{\delta \rho(\mathbf{r})} \mathrm{d} \mathbf{r}^{\prime}=\eta .
$$

One can eventually use its second definition (see (4)) to prove the equalization principle:

$$
\begin{aligned}
\eta(\mathbf{r}) & =\frac{\partial \mu(\mathbf{r})}{\partial N}=\int \frac{\delta \mu(\mathbf{r})}{\delta \rho\left(\mathbf{r}^{\prime}\right)} \frac{\partial \rho\left(\mathbf{r}^{\prime}\right)}{\partial N} \mathrm{~d} \mathbf{r}^{\prime} \equiv \int \eta\left(\mathbf{r}, \mathbf{r}^{\prime}\right) f\left(\mathbf{r}^{\prime}\right) \mathrm{d} \mathbf{r}^{\prime}, \\
& =\eta \iint \eta\left(\mathbf{r}, \mathbf{r}^{\prime}\right) \sigma\left(\mathbf{r}^{\prime}, \mathbf{r}^{\prime \prime}\right) \mathrm{d} \mathbf{r}^{\prime} \mathrm{d} \mathbf{r}^{\prime \prime}=\eta \int \delta\left(\mathbf{r}-\mathbf{r}^{\prime \prime}\right) \mathrm{d} \mathbf{r}^{\prime \prime}=\eta,
\end{aligned}
$$

where $\eta\left(\mathbf{r}, \mathbf{r}^{\prime}\right)$ is a hardness kernel, $f(\mathbf{r})$ is the Fukui function or the normalized local softness $(f(\mathbf{r}) \equiv$ $s(\mathbf{r}) / S) .{ }^{8}$ Local softness can be obtained by integrating softness kernel: $s(\mathbf{r})=\int \sigma\left(\mathbf{r}, \mathbf{r}^{\prime}\right) \mathrm{d} \mathbf{r}^{\prime}$, which is the inverse of the hardness kernel. The global softness is the inverse of the global hardness $(S=1 / \eta){ }^{8}$ The proof that $\int \eta\left(\mathbf{r}, \mathbf{r}^{\prime}\right) f\left(\mathbf{r}^{\prime}\right) \mathrm{d} \mathbf{r}^{\prime}$ was also given by Senet ${ }^{20}$ without referring to the reciprocal kernel. Using the chain rule transformation one can write the global hardness as $\eta=\int f(\mathbf{r}) \eta\left(\mathbf{r}, \mathbf{r}^{\prime}\right) f\left(\mathbf{r}^{\prime}\right) \mathrm{d} \mathbf{r d} \mathbf{r}^{\prime}$, therefore in the literature one can find some other definitions of the local hardness $\int \eta\left(\mathbf{r}, \mathbf{r}^{\prime}\right) g\left(\mathbf{r}^{\prime}\right) \mathrm{d} \mathbf{r}^{\prime},{ }^{8,23}$ where $g\left(\mathbf{r}^{\prime}\right)$ is a unity normalized function. Notice, that the alternative definitions lead to the local hardnesses that are not equalized throughout the space and can be considered as a reactivity indicators. Of course, its averaging by the Fukui function leads to the same value of the global hardness. There is no such an ambiguity for the chemical potential that is always equalized for equilibrium electron density.

In order to prove the equalization equations up to the infinite order, let us introduce the generalized global $n$-potential

$$
\mu^{(n)} \equiv \frac{\partial \mu^{(n-1)}}{\partial N},
$$

and its "local" equivalent

$$
\mu^{(n)}(\mathbf{r}) \equiv \frac{\delta \mu^{(n-1)}}{\delta \rho(\mathbf{r})}=\frac{\partial \mu^{(n-1)}(\mathbf{r})}{\partial N}
$$

As previously, the functional derivatives are evaluated for equilibrium density. Of course, $n=1(n=2)$ corresponds to chemical potential (hardness) and $\mu(0) \equiv E$. We further assume that $n$-potential fulfills the equalization equation,

$$
\mu^{(n)}=\mu^{(n)}(\mathbf{r}) .
$$

Thus, we have to prove that the above equation is valid for $(n+1)$-potential. The proof is straightforward if we start from definition of $(n+1)$-potential in "local" representation: 


$$
\begin{aligned}
\mu^{(n+1)}(\mathbf{r}) & =\frac{\delta \mu^{(n)}}{\delta \rho(\mathbf{r})}=\frac{\delta}{\delta \rho(\mathbf{r})} \frac{\partial \mu^{(n-1)}}{\partial N}=\frac{\partial}{\partial N} \frac{\delta \mu^{(n-1)}}{\delta \rho(\mathbf{r})} \\
& =\frac{\partial \mu^{(n)}(\mathbf{r})}{\partial N}=\frac{\partial \mu^{(n)}}{\partial N}=\mu^{(n+1)} .
\end{aligned}
$$

In the above derivation we have used Maxwell relation, definition of global $n$-potential and the assumption given in (8).

\section{Constraint equilibrium}

In this section we consider the constraint equilibrium case. Now, the whole reactive system $\mathrm{M}$ is divided into two mutually closed reactants, let say A and $\mathrm{B} .{ }^{12}$ Here, we assume that the total density is the sum of reactant densities: $\rho(\mathbf{r})=\rho_{A}(\mathbf{r})+\rho_{B}(\mathbf{r})$. Such partitioning is a common practice in energy decomposition schemes ${ }^{24-27}$ when one is interested in computing a given energy term and in linear scaling methods. ${ }^{28-31}$ We further assume that both reactants are closed to each other, but however, are open to their own electron reservoirs. Such a situation can be schematically represented as follows,

$$
M=\left(R_{\mathrm{A}} \mathrm{MA} \mid B \mathrm{M} R_{\mathrm{B}}\right) .
$$

Here, $R_{A}$ and $R_{B}$ are the reactant electron reservoirs. Solid vertical line indicates that the electron flow from B (a base) to A (an acid) is forbidden while the dotted one indicates that the charge flow between a given subsystem and its reservoir is allowed. In other words, the electron densities of reactants are relaxed; there are no other additional constraints on charge reorganization except that on charge transfer between reactants. One can eventually consider other hypothetical (non-equilibrium) situations: $M=\left(R_{A} \mathrm{M}\right.$ $A \mid B), M=\left(A \mid B M R_{B}\right)$, and $M=(A \mid B)$. If the reactant is not opened to its electron reservoir, it means that its electron density is frozen. Thus, the first partitioning describes the relaxed A and frozen $\mathrm{B}$, second the opposite situation, while in third one the electron densities of both reactants are frozen. Such hypothetical partitionings are very common in computational chemistry, e.g. energy partitioning schemes, ${ }^{24-27}$ the buffer (localized) zone between quantum and classical systems; ${ }^{32}$ successive freezing of subsystems electron densities in the elongation method, ${ }^{30,31}$ fragment-type calculations in other linear scaling methods ${ }^{28,29}$ etc.
As in part 2, we first concentrate on the equalization equations involving the reactant chemical potentials: ${ }^{12}$

$$
\begin{gathered}
\mu_{\mathrm{A}} \equiv\left(\partial E / \partial N_{\mathrm{A}}\right)_{N_{\mathrm{B}}} \quad \mu_{\mathrm{B}} \equiv\left(\partial E / \partial N_{\mathrm{B}}\right)_{N_{\mathrm{A}}}, \\
\mu_{\mathrm{A}}(\mathbf{r}) \equiv\left[\delta E / \delta \rho_{\mathrm{A}}(\mathbf{r})\right]_{N_{\mathrm{B}}}, \quad \mu_{\mathrm{B}}(\mathbf{r}) \equiv\left[\delta E / \delta \rho_{\mathrm{B}}(\mathbf{r})\right]_{N_{\mathrm{A}}},
\end{gathered}
$$

and hardnesses: ${ }^{12}$

$$
\begin{aligned}
\eta_{\mathrm{AA}} & \equiv\left(\partial \mu_{\mathrm{A}} / \partial N_{\mathrm{A}}\right)_{N_{\mathrm{B}}}, \eta_{\mathrm{BB}} \equiv\left(\partial \mu_{\mathrm{B}} / \partial N_{\mathrm{B}}\right)_{N_{\mathrm{A}}}, \\
\eta_{\mathrm{AB}} & \equiv\left(\partial \mu_{\mathrm{B}} / \partial N_{\mathrm{A}}\right)_{N_{\mathrm{B}}}, \\
\eta_{\mathrm{AA}}(\mathbf{r}) & \equiv\left[\delta \mu_{\mathrm{A}} / \delta \rho_{\mathrm{A}}(\mathbf{r})\right]_{N_{\mathrm{B}}}, \eta_{\mathrm{BB}}(\mathbf{r}) \equiv\left[\delta \mu_{\mathrm{B}} / \delta \rho_{\mathrm{B}}(\mathbf{r})\right]_{N_{\mathrm{A}}}, \\
\eta_{\mathrm{AB}}(\mathbf{r}) & \equiv\left[\delta \mu_{\mathrm{B}} / \delta \rho_{\mathrm{A}}(\mathbf{r})\right]_{N_{\mathrm{B}}} .
\end{aligned}
$$

Here, $N_{\mathrm{A}} / N_{\mathrm{B}}$ are the reactant electron populations. The mixed hardness derivatives $\eta_{\mathrm{A}, \mathrm{B}}$ and $\eta_{\mathrm{B}, \mathrm{A}}$ or $\eta_{\mathrm{A}, \mathrm{B}}(\mathbf{r})$ and $\eta_{\mathrm{B}, \mathrm{A}}(\mathbf{r})$ are equal due to the Maxwell relation. The functional derivatives in (12) and (14) are evaluated for equilibrium reactant densities. Finally, we give the general proof, again using the mathematical induction.

Let us first consider chemical potential equalization equations. Using the chain rule transformation $\sum_{X}\left(\partial / \partial N_{X}\right)\left(\delta N_{X} / \delta \rho_{A}(\mathbf{r})\right)$, where $X=\mathrm{A}$ and $\mathrm{B}$, one can easy verify that

$$
\begin{aligned}
\mu_{\mathrm{A}}(\mathbf{r}) \equiv & \left(\frac{\delta E}{\delta \rho_{\mathrm{A}}(\mathbf{r})}\right)=\frac{\partial E}{\partial N_{\mathrm{A}}} \frac{\delta N_{\mathrm{A}}}{\delta \rho_{\mathrm{A}}(\mathbf{r})} \\
& +\frac{\partial E}{\partial N_{\mathrm{B}}} \frac{\delta N_{\mathrm{B}}}{\delta \rho_{\mathrm{A}}(\mathbf{r})}=\mu_{\mathrm{A}} \int \frac{\delta \rho_{\mathrm{A}}\left(\mathbf{r}^{\prime}\right)}{\delta \rho_{\mathrm{A}}(\mathbf{r})} \mathrm{d} \mathbf{r}^{\prime}=\mu_{\mathrm{A}} .
\end{aligned}
$$

The last term disappears since electron perturbation in A does not change the number of electrons in B. In the same way, the chemical potential equalization for reactant B can be shown. The proof for hardnesses is almost identical, therefore we present here the equalization for the off-diagonal coupling hardness:

$$
\begin{aligned}
\eta_{\mathrm{BA}}(\mathbf{r}) & \equiv\left[\delta \mu_{\mathrm{A}} / \delta \rho_{\mathrm{B}}(\mathbf{r})\right]=\frac{\partial \mu_{\mathrm{A}}}{\partial N_{\mathrm{A}}} \frac{\delta N_{\mathrm{A}}}{\delta \rho_{\mathrm{B}}(\mathbf{r})}+\frac{\partial \mu_{\mathrm{A}}}{\partial N_{\mathrm{B}}} \frac{\delta N_{\mathrm{B}}}{\delta \rho_{\mathrm{B}}(\mathbf{r})} \\
& =\eta_{\mathrm{AA}} \int \frac{\delta \rho_{\mathrm{A}}\left(\mathbf{r}^{\prime}\right)}{\delta \rho_{\mathrm{B}}(\mathbf{r})} \mathrm{d} \mathbf{r}^{\prime}+\eta_{\mathrm{BA}} \int \frac{\delta \rho_{\mathrm{B}}\left(\mathbf{r}^{\prime}\right)}{\delta \rho_{\mathrm{B}}(\mathbf{r})} \mathrm{d} \mathbf{r}^{\prime}=\eta_{\mathrm{BA}}
\end{aligned}
$$


Knowledge of the reactant chemical potential and hardnesses allows one to compute the charge-transfer (CT) contribution to the overall interaction energy in the quadratic approximation:

$$
E_{\mathrm{CT}}=-\left(\mu_{\mathrm{A}}-\mu_{\mathrm{B}}\right)^{2} / 2\left(\eta_{\mathrm{AA}}+\eta_{\mathrm{BB}}-2 \eta_{\mathrm{AB}}\right) \equiv-\mu_{\mathrm{CT}}^{2} / 2 \eta_{\mathrm{CT}} \text {. }
$$

The chemical potential difference (CT chemical potential) is a driving force restoring the global equilibrium in the system while the $\mathrm{CT}$ hardness reflects the resistance of the charge distribution. We would like to mention here that it is the only contribution to the interaction energy that depends regularly on the donor/acceptor properties of the interacting species. $^{27}$

Let us now introduce the general $n$-potentials:

$$
\begin{aligned}
& \mu_{\alpha \mathrm{A}}^{(n)} \equiv \frac{\partial \mu_{\alpha}^{(n-1)}}{\partial N_{\mathrm{A}}}, \mu_{\alpha \mathrm{B}}^{(n)} \equiv \frac{\partial \mu_{\alpha}^{(n-1)}}{\partial N_{\mathrm{B}}} \\
& \mu_{\alpha \mathrm{A}}^{(n)}(\mathbf{r}) \equiv \frac{\delta \mu_{\alpha}^{(n-1)}}{\delta \rho_{\mathrm{A}}(\mathbf{r})}, \quad \mu_{\alpha \mathrm{B}}^{(n)}(\mathbf{r}) \equiv \frac{\delta \mu_{\alpha}^{(n-1)}}{\delta \rho_{\mathrm{B}}(\mathbf{r})}
\end{aligned}
$$

Here, $\alpha$ is a sequence of $n-1$ letters A or B. For example, $n$ equal to 1 corresponds to chemical potential, 2 to hardness data. We have just shown that equalization takes place for $n$ equal to one. Let us assume that

$$
\mu_{\alpha}^{(n)}=\mu_{\alpha}^{(n)}(\mathbf{r})
$$

Now, for convenience $\alpha$ is a sequence of $n$ letters A and/or B. We will show that this equality is fulfilled for $(n+1)$-potential:

$$
\begin{aligned}
\mu_{\alpha \mathrm{A}}^{(n+1)}(\mathbf{r}) & =\frac{\delta \mu_{\alpha}^{(n)}}{\delta \rho_{\mathrm{A}}(\mathbf{r})}=\frac{\delta}{\delta \rho_{\mathrm{A}}(\mathbf{r})} \frac{\partial \mu_{\beta}^{(n-1)}}{\partial N_{X}}=\frac{\partial}{\partial N_{X}} \frac{\delta \mu_{\beta}^{(n-1)}}{\delta \rho_{\mathrm{A}}(\mathbf{r})} \\
& =\frac{\partial \mu_{\beta \mathrm{A}}^{(n)}(\mathbf{r})}{\partial N_{X}}=\frac{\partial \mu_{\beta \mathrm{A}}^{(n)}}{\partial N_{X}}=\mu_{\alpha \mathrm{A}}^{(n+1)} .
\end{aligned}
$$

Here, $\beta=\alpha-X$; in other words $\beta$ is $(n-1)$-sequence of letters obtained from $\alpha$ by disregarding $X=\mathrm{A}$ or B. Notice that the quantities that differ only by permutation of indices are equal due to the Maxwell relation (e.g., $\left.\mu_{\mathrm{AAAB}}^{(4)}=\mu_{\mathrm{AABA}}^{(4)}=\mu_{\mathrm{ABAA}}^{(4)}=\mu_{\mathrm{BAAA}}^{(4)}\right)$.

\section{Conclusions}

In this paper, we have demonstrated that the population derivatives of the system energy in reactant resolution (mutually polarized interacting reactants) are equalized throughout the space up to the infinite order in the perturbation expansion. For example, the first- and second-order derivatives are the reactant chemical potentials and the hardness data, respectively. They are the most frequently explored quantities in the theory of chemical reactivity. They define the charge-transfer stabilization energy which is often a starting point for analyzing different reactivity descriptors and matching rules. The higher population derivatives can be important in strongly coupled reactants when the quadratic expansion of the system energy is questionable.

The direct consequence of the equalization equation is that the "canonically" coupled quantities or the "inverse" quantities to given population derivatives are additive. The number of electrons in molecular fragments and the reactant softness data are the best known examples.

The equalization equations can be used to test the distortion from the global equilibrium in linear scaling methods such as elongation, divide and conquer, and fragment molecular methods. In all these schemes the system global energy is computed by assuming a certain fragmentation technique. Therefore, one can established a well-defined hierarchy of corrections to the system energy resulting from the fact that charge distribution does not correspond to the global equilibrium.

\section{References}

1. Hohenberg P and Kohn W 1964 Phys. Rev. B136 864

2. (a) Thomas L H 1927 Proc. Camb. Philos. Sci. 23 542; (b) Fermi E 1927 Rend. Accad. Lincei 6 602; (c) Dirac P A M 1930 Proc. Camb. Philos. Soc. 26376

3. Kohn W and Sham L J 1965 Phys. Rev. A140 1133

4. Mulliken R S 1934 J. Chem. Phys. 2782

5. Sanderson R T 1951 Science 114670

6. (a) Pearson R G 1967 Chem. Br. 3 103; (b) Pearson R G 1973 Hard and soft acids and bases (Stroudsburg: Dowden, Hutchinson, and Ross)

7. (a) Pearson R G 1987 J. Chem. Edu. 64 561; (b) Pearson R G 1993 Acc. Chem. Res. 26250

8. Parr R G and Yang W 1989 Density functional theory of atoms and molecules (New York: Oxford University Press)

9. Parr R G, Donnelly R A, Levy M and Palke W E 1978 J. Chem. Phys. 683801 
10. Parr R G and Pearson R G 1883 J. Am. Chem. Soc. 1057512

11. (a) Parr R G and Yang W $1984 \mathrm{~J}$. Am. Chem. Soc. 106 4049; (b) Ayers P W and Parr R G $2000 \mathrm{~J}$. Am. Chem. Soc. 1222010

12. Nalewajski R F and Korchowiec J 1997 Charge sensitivity approach to electronic structure and chemical reactivity (Advanced series in physical chemistry) (Singapore: World Scientific) vol 8

13. Geerlings P, De Proft F and Langenaeker W 2003 Chem. Rev. 1031793

14. (a) Yang W and Mortier W 1986 J. Am. Chem. Soc. 108 5708; (b) Korchowiec J and Uchimaru T $1998 \mathrm{~J}$. Chem. Phys. A102 10167; (c) Balawender R and Komorowski L 1998 J. Chem. Phys. 109 5203; (d) Michalak A, De Proft F, Geerlings P and Nalewajski R F 1999 J. Phys. Chem. A103 762; (e) Contreras R R, Fuentealba P, Galvan M and Perez P 1999 Chem. Phys. Lett. 304 405; (f) Fuentealba P, Perez P and Contreras R 2000 J. Chem. Phys. 113 2544; (g) Ayers P W, Morrison R C and Roy R K 2002 J. Chem. Phys. 116 8731; (h) Bulat F A, Chamorro E, Fuentealba $\mathrm{P}$ and Toro-Labbe A 2004 J. Phys. Chem. A108 342

15. Gazquez J L and Mendez F J 1994 J. Phys. Chem. 98 4591; (b) Damoun S, Van de Woude G, Mendez F and Geerlings P 1997 J. Phys. Chem. A101 886

16. (a) Parr R G, Szentpaly L and Liu S 1999 J. Am. Chem. Soc. 121 1922; (b) Szentpaly L 2000 Proc. Natl. Acad. Sci. USA 95 11578; (c) Chattaraj P K and Maiti B 2001 J. Phys. Chem. A105 169; (d) Chattaraj P K, Perez P, Zevallos J and Toro-Labbe A $2001 \mathrm{~J}$. Phys. Chem. A105 4272; (e) Chomorro E, Chattaraj P K and Fuentealba P 2003 J. Phys. Chem. A107 7068

17. Chattaraj P K, Maiti B and Sarkar U 2003 J. Chem. Phys. A107 4973

18. Harbola M K, Chattaraj P K and Parr R G 1991 Israel J. Chem. 31395

19. Fuentealba P and Parr R G 1991 J. Chem. Phys. 94 5559

20. Senet P 1996 J. Chem. Phys. 1056471

21. Chamorro E, Fuentealba P and Contreras R $2001 \mathrm{~J}$. Chem. Phys. 1156822

22. (a) Komorowski L and Ordon P 2003 J. Mol. Struct. (Theochem.) 630 25; (b) Komorowski L and Ordon P 2004 Int. J. Quantum. Chem. 99 153; (c) Ordon P and Komorowski L 2005 Int. J. Quantum Chem. 101 703
23. (a) Meneses L, Tiznado W, Contreras R and Fuentealba P 2004 Chem. Phys. Lett. 383181

24. (a) Morokuma K 1971 J. Chem. Phys. 55 1237; (b) Iwata S and Morokuma K 1973 J. Am. Chem. Soc. 95 7563; (c) Kitaura K and Morokuma K 1976 Int. J. Quantum Chem. 10 325; (d) Morokuma K 1977 Acc. Chem. Res. 10294

25. (a) Stevens W J and Fink W H 1987 Chem. Phys. Lett. 139 15; (b) Chen W and Gordon M S 1996 J. Phys. Chem. 10014316

26. (a) Sokalski W A, Roszak S, Hariharan PC and Kaufman J J 1983 Int. J. Quantum Chem. 23 847; (b) Sokalski W A, Roszak S M and Pecul K 1988 Chem. Phys. Lett. 153 153; (c) Sokalski W A and Roszak S 1991 J. Mol. Struct. (Theochem.) 234387

27. (a) Korchowiec J and Uchimaru T 1998 J. Phys. Chem. A102 6682; (b) Korchowiec J and Uchimaru T 2000 J. Chem. Phys. 112 1623; (c) Korchowiec J, Chandra A K and Uchimaru T 2001 J. Mol. Struct. (Theochem.) 572 193; (d) Korchowiec J 2003 J. Mol. Struct. (Theochem.) 663 175; (e) Korchowiec J 2005 Int. J. Quantum. Chem. 101714

28. (a) Yang W 1991 Phys. Rev. Lett. 66 447; (b) Yang W 1991 Phys. Rev. A44 7823; (c) Yang W and Lee T S 1995 J. Chem. Phys. 1035674

29. (a) Kitaura K, Sawai T, Asada T, Nakano $T$ and Uebayasi M 1999 Chem. Phys. Lett. 312 319; (b) Kitaura K, Ikeo E, Asada T, Nakano T and Uebayasi M 1999 Chem. Phys. Lett. 313 701; (c) Fedorov D G, Olson R M, Kitaura K, Gordon M S and Koseki S 2004 J. Comput. Chem. 25872

30. (a) Imamura A, Aoki Y and Maekawa K $1991 J$. Chem. Phys. 95 5419; (b) Aoki Y and Imamura A 1992 J. Chem. Phys. 978432

31. (a) Gu F L, Aoki Y, Korchowiec J, Imamura A and Kirtman B 2004 J. Chem. Phys. 121 10385; (b) Korchowiec J, Gu F L, Imamura A, Kirtman B and Aoki Y 2005 Int. J. Quant, Chem. 102 785; (c) Korchowiec J, Gu F L and Aoki Y (ICCMSE2004 volume) (submitted)

32. (a) Jensen J H, Day P N, Gordon M S, Basch H, Cohen D, Garmer D R, Krauss M and Stevens W J 1994 Modeling the hydrogen bond (ed.) D A Smith ACS Symposium Series 569 pp 139-151; (b) Chen W and Gordon M S 1996 J. Chem. Phys. 105 11081; (c) Merrill G N and Gordon M S 1998 J. Phys. Chem. A102 2650; (d) Webb S P and Gordon M S 1999 J. Phys. Chem. A103 1265 